\title{
The calculation of the supercritical boiler's life expectancy used for frequency regulation
}

\author{
Haiyang $\mathrm{Yu}^{1, a}$, Runxin Chen ${ }^{2, a}$, Yufeng $\mathrm{Guo}^{2, \mathrm{~b}}$ \\ ${ }^{1}$ State Grid Heilongjiang Electric Power Research Institute, Heilongjiang Harbin, China 150030 \\ ${ }^{2}$ Harbin Institute of Technology of Electrical engineering and Automation Institute, Heilongjiang \\ Harbin, China 150001 \\ a124963564@qq.com, ${ }^{b}$ runxin_hit@163.com, ’guoyufeng@hit.edu.cn
}

\begin{abstract}
Keywords: Starting water separator, High temperature super-heater outlet header, Life expectancy, Rain flow method, Supercritical boiler

Abstract:During operation, starting water separator's main stress is closely related to the stress of water wall outlets. Starting water separator suffers a long period of stress characterized as alternating stress and low frequency, which suggests that the loss is mainly in the form of fatigue. In order to analyze these three factors, the method of the rain flow is presented here. Combining US ASME fatigue curve, life expectancy of starting water separator can be calculated. Then analyze the high temperature super-heater outlet's stress, which is related to super-heater's temperature and pressure. Using the time parameter method and life loss fraction method, life expectancy of high temperature super-heater outlet can be calculated. This calculation method can be used to analyze the frequency regulation.
\end{abstract}

\section{Introduction}

With the growing demand of electricity users, Grid-side on unit peaking capacity requirements continue to increase, which means that actual operating conditions of the pressure-bearing part of utility boiler subject to may exceed the scope of conventional design. ${ }^{[1]}$ Meanwhile, pressure-bearing part usually works under the condition of more than $600^{\circ} \mathrm{C}$ and $20 \mathrm{MPa}$, which is seriously severe. After 8 years of follow-up survey of 70 thermal power station in Japan, pressure-bearing part accounted for $70 \%$ of the total damage of boiler components. ${ }^{[2]}$ According to reference [3], starting water separator and high temperature super-heater outlet are the most important but also the easiest broken pressure-bearing parts in supercritical boiler.

The main reason for the failure of the starting water separator is the creep loss caused by high temperature during thousands of load changes and countless hot start. ${ }^{[4]}$ In the other hand, creep life loss is the main loss form of the high temperature super-heater outlet. ${ }^{[5]}$ Creep life prediction method is now based mainly on the time parameter method. Larson-Miller formula is the most famous time parameter extrapolation method. ${ }^{[6]}$

\section{The Calculation of total stress of the starting water separator}

For an unperforated cylindrical body member, the internal pressure caused by the mechanical stress can be expressed as Eq. 1:

$$
\sigma_{\mathrm{m}}=p \frac{\beta^{2}+1}{\beta^{2}-1}
$$

Analytical and experimental study of mechanical stress distribution shows that, there exist obvious stress concentration phenomenon in steam separator. Here we introduce the concept of mechanical stress concentration factor $k_{\mathrm{j}}$. Different regions have different physical factors. So the value of $k_{\mathrm{j}}$ changes accordingly. The expression of $k_{\mathrm{j}}$ is shown as Eq. 2: 


$$
k_{\mathrm{j}}=\frac{\sigma_{\max }}{\sigma}
$$

ANYSY stress analysis shows that, the value of steam separator's maximum mechanical stress concentration coefficient is basically stabilized at around 3.2. The most dangerous point is located in the long axis of the ellipse hole of the intersecting line for the inner wall and soda introduction pipe of the separator. Mechanical stress multiplied by the mechanical stress concentration factor, is the steam separator's total pressure.

\section{The Calculation method of fatigue wear and tear}

In the supercritical boiler, steam separator is characterized by high peak stress but few cycles. Therefore it's more prone to produce low cycle fatigue wear and tear. This article uses the rain flow method to calculate both the times of cycle and the amplitude of steam separator's total stress.

Specific detection of rain flow method has four steps. The first step requires the program to identify peaks or troughs. Check all the data in chronological order, then select three adjacent points that satisfy Eq.3 or Eq.4 and exclude the remaining points.

$$
\begin{gathered}
\left\{\begin{array}{l}
\sigma_{i-1}<\sigma_{i} \\
\sigma_{i}>\sigma_{i+1}
\end{array}\right. \\
\left\{\begin{array}{l}
\sigma_{i-1}>\sigma_{i} \\
\sigma_{i}<\sigma_{i+1}
\end{array}\right.
\end{gathered}
$$

After selecting out all the peaks and troughs, compare the value of four adjacent points $\sigma_{i-1}$ 、 $\sigma_{i}$ 、 $\sigma_{i+1} 、 \sigma_{i+2}$. If these values satisfy Eq. 5 or Eq. 6 , then point $\mathrm{i}, \mathrm{i}+1$, and $\mathrm{i}^{*}$ constitute a full cycle, as shown in Fig. 1. Half the difference between the two points $i$ and $i+1$ is the full cycle's stress amplitude.
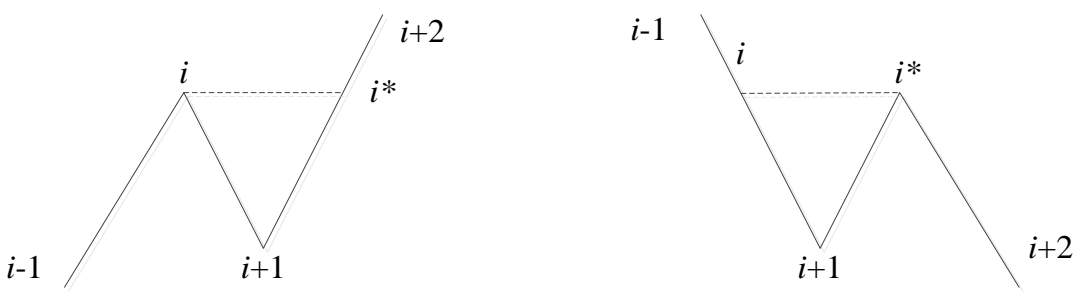

Fig. 1 four points comparison method

$$
\begin{gathered}
\left\{\begin{array}{l}
\sigma_{i-1}<\sigma_{i} \\
\sigma_{i-1} \leq \sigma_{i+1} \\
\sigma_{i+2} \geq \sigma_{i+1}
\end{array}\right. \\
\left\{\begin{array}{l}
\sigma_{i-1}>\sigma_{i} \\
\sigma_{i-1} \geq \sigma_{i+1} \\
\sigma_{i+2} \leq \sigma_{i}
\end{array}\right.
\end{gathered}
$$

Then check those points that satisfy Eq. 3 and Eq. 4, but not satisfy Eq. 5 or Eq. 6, take two adjacent points as a half cycle. Semi-cyclic stress amplitude is equal to half the difference between 
these two points, and calculate the times of half cycle as $N_{i k}$. This article use the American ASME design fatigue curve. Using the rain flow method to get the stress amplitude, then determine the stress is under full cycle or half cycle, find the ASME design fatigue curve to get the corresponding cyclic number $N$. Thus, fatigue life loss $\mathrm{F}$ can be calculated as follows:

$$
F=\sum_{i=1}^{n} \frac{1}{N_{\mathrm{in}}}+\sum_{i=1}^{k} \frac{0.5}{N_{\mathrm{ik}}}
$$

\section{The Calculation method of creep life loss}

Researchers propose a method which is called time-temperature parameter method by referring to the high-temperature but short-term experiments. This method assumes that the creep rate follows Eq. 8 at a given stress.

$$
v=A \mathrm{e}^{-\frac{Q}{R T}}
$$

Larson-Miller method is a use of time-temperature parameter method. Using the least square fit method to process the test data which are obtained by changing the stress value, the different values of $C_{i}$ can be calculated. Creep-off time can be written as a function of temperature $T$ and stress $\sigma$ :

$$
\lg \tau=-C+\frac{C_{0}}{T}+\frac{C_{1}}{T} \lg \sigma+\frac{C_{2}}{T} \lg ^{2} \sigma+\frac{C_{3}}{T} \lg ^{3} \sigma
$$

After operating for some time $\tau$ in a particular stress and temperature, the life loss of this material can be expressed as $\frac{\tau}{\tau_{\mathrm{r}}}$, which is called the life-loss factor. $\tau_{\mathrm{r}}$ shows the material's fracture time under specific stress and temperature. In practice, boiler's temperature and pressure is changing all the time. Then take the fractional life loss $\phi_{\mathrm{r}}$ as the sum of life loss under various conditions. When $\phi_{\mathrm{r}}$ reaches 1 , that is, the end of life.

\section{Numerical examples}

By simulating the actual daily load of power grid, the stress that starting water separator suffered is showed in Fig. 2. The stress that high temperature super-heater outlet header suffered is showed in Fig. 3.

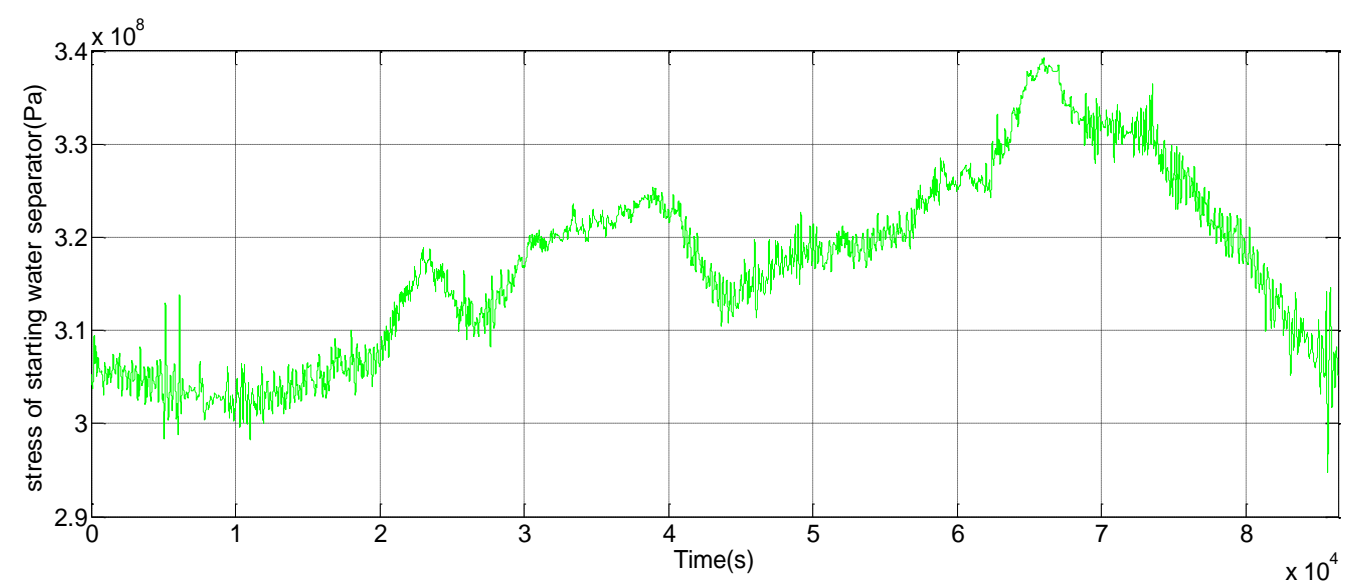

Fig. 2 The stress of starting water separator 


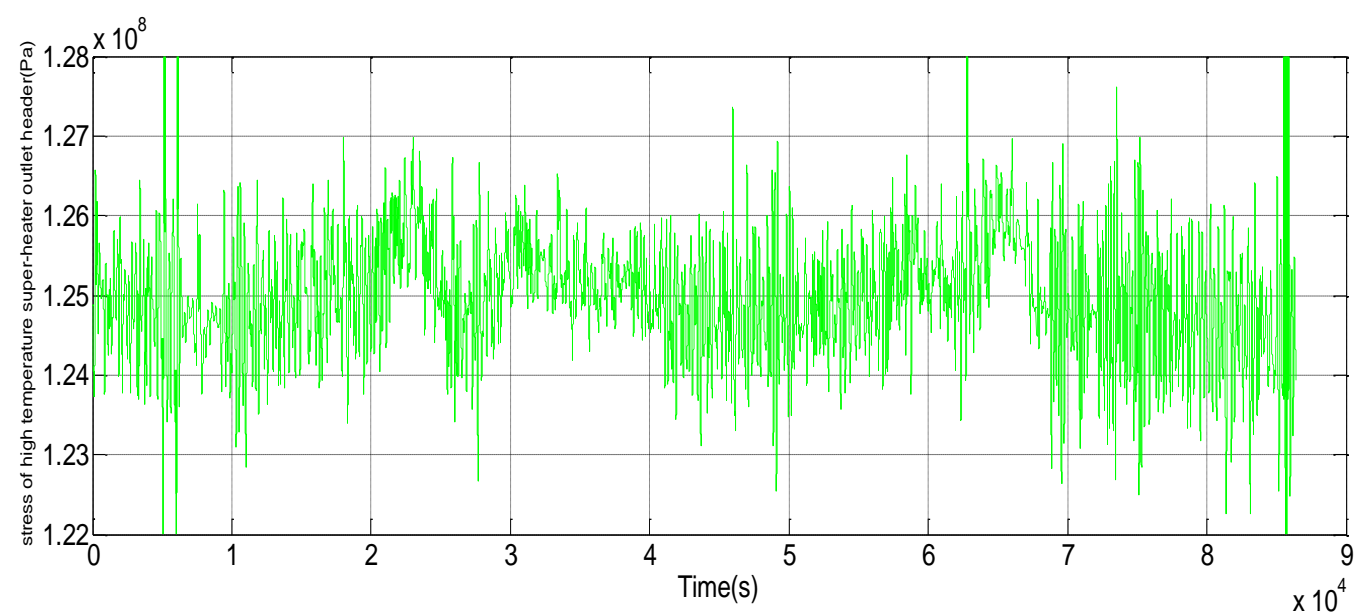

Fig. 3 The stress of high temperature super-heater outlet header

According to the rain flow method, the life expectancy of high temperature is 34.1609 years. The number of full cycle is 2146 , while the number of half cycle is 6 . According to the time parameter method, the life expectancy of high temperature is 26.5535 years.

\section{Conclusions}

Suffered from different types of stress, form of these pressure parts' life loss varies. Starting water separator bears fatigue wear and tear, the calculation of which is closely related to the mechanical stress caused by dramatic changes in vapor pressure. Using the rain flow method and US ASME fatigue curves, the life expectancy of starting water separator can be figured out. Meanwhile, high temperature super-heater outlet header bears creep life loss, the calculation of which is closely related to both thermal stress and mechanical stress. Using the Larson-Miller method and life loss fraction method, the life expectancy of high temperature super-heater outlet header can be figured out.

\section{Acknowledgements}

This work was financially supported by National Key Basic Research Program of China (973 Program) (2012CB215201) and the National Natural Science Foundation of China (Grant No.51676054).

\section{References}

[1] Li Jianwei. Service life analysis for the thick-wall components of the $600 \mathrm{MW}$ supercritical boiler. Baoding: North China Electric Power University, 2009.

[2] Lu Gaoming. Analysis of stress and calculation of fatigue life in the procedure of starting up for 300 MW boiler drum. Harbin: Harbin Institute of Technology, 2007.

[3] DING Yangjun, SHENG Deren, CHEN Jianhong. Optimization of start-up process in combined cycle steam turbine of a power plant. Proceedings of the CSEE, 2013, 33(2):9-15.

[4] Liu Tong, Shi Fei, Sun Baomin. Stress analysis and simulation of a supercritical boiler's starting water separator. Power Engineering, 2007, 27(6):868-871.

[5] Li Yaojun. Development and application of service-life management technology for important parts of thermal power plants. Thermal Power Generation, 2003, 32(5):9-12.

[6] Wang Aimin, Lin Hongshu. Method for high-temperature creep-fatigue damage analysis and life prediction based on elastic analysis. Pressure vessel Technology, 1999,16(1):18-24. 